\title{
A litmus test for harmonized mosquito monitoring across Europe and Africa
}

Ignazio Graziosi ${ }^{1}$, Carles Aranda ${ }^{2,11}$, Fabrizio Balestrino ${ }^{3}$, Romeo Bellini ${ }^{3}$, Núria Busquets ${ }^{2}$, Mammadou Coulibaly ${ }^{4}$, Andrea Crisanti ${ }^{5}$, Diawo Diallo ${ }^{6}$, Mawlouth Diallo $^{6}$, Alioune Gaye ${ }^{6}$, Moussa Guelbeogo ${ }^{7}$, Aleksandra Ignjatović-Ćupina ${ }^{8}$, Sebastián Napp ${ }^{2}$, Sagnon N'Falé 7 , Dušan Petrić $^{8}$, Paola Pollegioni ${ }^{9}$, Alekos Simoni ${ }^{1}$, Marija Zgomba ${ }^{8}$, Ruth Müller ${ }^{1,10}$

${ }^{1}$ Polo d'Innovazione di Genomica Genetica e Biologia (Polo GGB), Terni, Italy.

${ }^{2}$ IRTA, Centre de Recerca en Sanitat Animal (CReSA, IRTA-UAB), Campus de la Universitat Autònoma de Barcelona, 08193 Bellaterra, Spain.

${ }^{3}$ Centro Agricoltura Ambiente, Department of Sanitary Entomology and Zoology (EZS), Crevalcore, Bologna, Italy.

${ }^{4}$ Malaria Research and Training Centre, Université de Bamako, Bamako, Mali.

${ }^{5}$ Imperial College London, London, UK.

${ }^{6}$ Institut Pasteur de Dakar, Dakar, Senegal.

${ }^{7}$ Centre National de Recherche et de Formation sur le Paludisme (CNRFP), Ouagadougou, Burkina Faso.

${ }^{8}$ University of Novi Sad, Faculty of Agriculture, Novi Sad, Serbia.

${ }^{9}$ Consiglio Nazionale delle Ricerche, Istituto di Ricerca sugli Ecosistemi Terrestri (CNR-IRET), Porano, Terni.

${ }^{10}$ Institute of Tropical Medicine, Unit Entomology, Antwerp, Belgium.

${ }^{11}$ Servei de Control de Mosquits, Consell Comarcal del Baix Llobregat, Barcelona, Spain.

Keywords: mosquito surveillance, vector monitoring, larval production sites, mosquito breeding sites 
Abstract: The accelerating rate of outbreaks from mosquito borne diseases are urging the development of updated and effective tools for the surveillance of insect populations and their larval habitats. Harmonized field protocols help to build a comprehensive picture on species-specific vector ecology and generate important knowledge for implementing coordinated mosquito surveillance programs at regional scales and across continents. In this study, we test the efficiency and potential barriers of available harmonized protocols from earlier EU project VectorNet. As a kind of litmus test for such protocols, we specifically aim to capture the ecoregional variation of breeding site characteristics and population density of five mosquito vectors in Europe and Africa. As expected, the five species considered show different aquatic habitat preferences. Culex pipiens, Aedes albopictus in Europe and Ae. aegypti in Africa select breeding habitats within specific volume classes, while Anopheles gambiae and An. coluzzii may select breeding habitats based on seasonal availability. Population densities in aquatic habitats greatly varied across species and countries, but larval production sites of Ae. albopictus generate populations with higher ratio of pupae compared to the other species. This result underlines the fundamental ecological difference between the selected vector species disregarding of the ecoregion. Mean water temperatures had limited variation across species and higher among countries. Understanding the ecology of native and non-native mosquito vectors is key in evaluating transmission risks for diseases such as West Nile, chikungunya and dengue fevers, zika and malaria. The available harmonized field protocols are a valuable tool for achieving homogeneous mosquito surveillance in Europe and Africa. 


\section{Introduction}

The global trend of mosquito-borne diseases is dramatically rising, with recrudescence of some well-known pathogens and accelerating outbreaks of emerging diseases in multiple regions (Jones et al. 2008). mosquito-borne diseases are a major burden to human health, thus determining pervasive health and socio-economic costs to local communities (Gubler 1998). We are witnessing the rising of once neglected arboviral tropical diseases carried by native and invasive mosquitoes, namely dengue fever, Zika, chikungunya and West Nile, which are quickly becoming pandemic and pose a current threat for Europe and Africa (Benelli and Mehlhorne 2016, Weaver et al. 2018). Accelerated human mobility facilitates the movement of infected individuals (Sarma et al. 2018), global trade is intensifying introduction of non-native vectors, and changing climates and anthropization are creating suitable habitats for mosquitoes, thus magnifying proliferation and risk of transmission (Derraik and Slaney 2007). Furthermore, malaria incidence is locally increasing in high-risk areas of Africa and South America and resurging unexpectedly in Greece (Danis et al. 2011, Whitty and Ansah, 2019).

As a result of anthropogenic-driven mosquito proliferation and climatic adaptations we may expect the recrudescence of old and new infectious diseases vectored by several species of mosquitoes (Diptera: Culicidae) world-wide (Hotez 2016), including Culex and Aedes (Culicinae) and Anopheles (Anophelinae). Culex pipiens Linnaeus is a common mosquito with world-wide distribution, a competent vector for West Nile and Japanese encephalitis viruses which contributed to West Nile outbreaks in Southern Europe and North America (Wispelaere 2017, Marini et al. 2020). The highly invasive Aedes albopictus (Skuse) is expanding its non-native range in Europe thanks to exceptional ecological plasticity, and is increasingly alarming health organizations and surveillance networks for its ability to cause dengue, Zika and chikungunya outbreaks (Aranda et al. 2018, Giron et al. 2019, Javelle et al. 2019). Congeneric Ae. aegypti, the vector of yellow fever virus in Africa, is also competent for dengue, chikungunya and Zika viruses, thus magnifying infection risks in Europe, where the species was also detected (Akiner et al. 2016). Anopheles gambiae Giles and An. coluzzii Coetzee \& Wilkerson are the most widespread species causing malaria in Sub Saharan Africa, with the former preferring habitats in the African humid tropics and the latter dryer habitats, typically in the Sahel (Lanzaro and Lee 2013, Coetzee et al. 2013). 
Understanding the ecology of native and non-native mosquito vectors, and how habitat availability define their distribution, is key in evaluating transmission risks. Environmental parameters of mosquito breeding site (MBS) shape insect development and ultimately affect their vector competence. The productivity of MBS is determined by water temperature, volume of breeding environment, predator density or presence of organic matter (Bevins 2008, Okech et al. 2007, Schneider et al. 2011). The variation of the productivity of MBS within ecoregions is still unclear (Pascoe et al. 2019); though the characterization of such variations would add fundamental knowledge on population dynamics and structures (Okogun et al. 2003, Vallorani et al. 2015).

The success of managing emerging and resurging mosquito-borne diseases relies on designing effective integrated vector management (IVM) tools, which include active vector monitoring (Fernandes et al. 2018). The effectiveness and reliability of pan-regional systems for vector monitoring requires the development of homogeneous methodologies. Recent EU research networks (Vector Net, AIM Cost) aimed to standardize procedures for the surveillance of arthropod vectors in Europe in order to provide standardized datasets (Versteirt et al. 2017, Medlock et al. 2018). Suggestions on the use of harmonised methods, equipment and field protocols for sampling have been published (ECDC and EFSA 2018). However, a litmus test of such protocols is needed to evaluate the realistic potential of methodological standardization and challenges in different ecoregional settings (Vernick 2017).

Within the framework of the EU funded InfraVec2 consortium, we tested the efficiency and potential barriers of available harmonized protocols from earlier EU project VectorNet. As a kind of litmus test for such protocols, we specifically aimed to capture the ecoregional variation of MBS and population densities of five target mosquito vectors in Europe and Africa.

\section{Material and methods}

We conducted a 6-country study describing the variation in natural vector larval populations to test harmonized field protocols and assessed regional variation of MBS parameters for five mosquito species: (1) Cx. pipiens in Spain, Italy and Serbia, (2) Ae. albopictus in Spain 
and Italy, (3) Ae. aegypti in Senegal, (4) An. gambiae in Senegal and Burkina Faso, and (5) An. coluzzii in Mali.

At first, we jointly developed standard operating procedures (SOP) for a MBS survey based on the VectorNet field protocols and guidelines from the European Centre for Disease Prevention and Control (ECDC and EFSA 2018). The agreed SOP was tested in all six countries: in Catalonia, Spain, in the Emilia Romagna region of Italy, in Novisad and Voivodina area in Serbia, Dakar and Kedougou province in Senegal, Ouagadougou and Banfora areas in Burkina Faso, and Bamako and Kati districts in Mali (Fig. 1). Potential MBS were surveyed in 2018 and 2019 during mosquito peak season (July to early August in Europe, late September to early November in Africa) except from Mali, which was surveyed at the end of the peak season (late December).

In order to systematically comprise MBS variation across different landscapes, we surveyed potential MBS in urban (city), sub-urban (suburbs), rural (cropland) and natural habitats (rivers, wetlands, woodlands). For each MBS (mosquito-positive site), geographic coordinates were collected using hand-held GPS devices when available, or smartphones, georeferenced and displayed using Google Earth, and data from the field harmonized using MS Excel. The volume of MBS was assessed by measuring or by estimating the approximate dimensions of the water container/body of water. MBS were assigned to 6 volume classes: 1 (<0.5 L), 2 (0.5-5.0 L), 3 (5-50 L), 4 (50-200 L), 5 (200-2000 L), 6 (>2000 L). The type of MBS was also noted (i.e. river, pond, puddle, pot, tire, or other). We measured water temperature in the tray immediately to avoid heating using hand-held electronic thermometers with accuracy between $0.1-0.5^{\circ} \mathrm{C}$.

In addition, we determined the mosquito population density and population structure in each MBS by using a dipper (or pipette for smaller breeding sites) to transfer $1 \mathrm{~L}$ of water from the site into a plastic tray (or all the water for breeding sites $<1 \mathrm{~L}$ ). We counted the number of young larvae (L1-L2), mature larvae (L3-4), and pupae (P) in the tray. Larvae and pupae were identified to species based on macro-morphology and habit. We also calculated the total number of mosquitoes $T=L 1-2+L 3-4+P$, and a pupal ratio $R=P / T$. For larger MBS, the water sampling and counting of larvae and pupae were replicated ten consecutive times. Means, standard deviations (STD), coefficient of variation (CV) and 
standard errors (SE) were calculated to assess variations of population density, population structure and MBS temperature.

\section{Results}

In total, 191 MBS were assessed (Table 1): Cx. pipiens was collected from a total of 69 MBS (3 countries, Europe), Ae. albopictus from 35 MBS (2 countries, Europe), Ae. aegypti from 19 MBS (1 country, Africa), An. gambiae from 50 MBS (2 countries, Africa) and An. coluzzii from 18 MBS (1 country, Africa). Exact locations of MBS are available for download at: https://infravec2.eu/mosquito-survey-data/. We detected species-specific preferences for certain physical shape and volume of MBS: $C x$. pipiens was preferentially found in canals, manholes, and streams, Ae. albopictus in flower pots or barrels used for common garden irrigation, Ae. aegypti in tires, An. gambiae in clay quarries and puddles, and An. coluzzii in puddles near river beds. The volume of MBS ranges from class 2 to 6 for $C x$. pipiens, 1 to 4 for Ae. albopictus, 1 to 3 for Ae. aegypti, 3 to 5 for An. gambiae, and only class 3 for An. coluzzii. The characteristics and volume classes of MBS preferred by $C_{x}$. pipiens, Ae. albopictus and An. gambiae slightly vary among countries (Table 2, Fig. 2). The lowest temperature values were found in MBS of $C x$. pipiens, and the highest in MBS of two Aedes species. The overall temperature in MBS varies only slightly across surveyed countries in Europe and Africa, ranging between 26.3 to $27.6{ }^{\circ} \mathrm{C}$ with an overall STD of 3.3 and CV of $12.5 \%$ (Table 3). The only exception is the mean temperature of the MBS of An. gambiae, which differs strongly between countries: in Burkina Faso the mean MBS temperature is $2^{\circ} \mathrm{C}$ cooler if compared to values of the respective MBS in Senegal $\left(26.1{ }^{\circ} \mathrm{C}\right.$ and $28.1{ }^{\circ} \mathrm{C}$ respectively, Fig. 3).

The population density is highly variable across species though Ae. albopictus shows lower densities and smaller coefficient of variation compared to the other four taxa. The highest total population densities across countries are produced by $C x$. pipiens. whereas its highest total density is reported from a waste container in Serbia ( $n=688$ per liter). For comparison, the highest population density for Ae. albopictus was detected in a scupper drain in Spain ( $n=114$ per liter). In Africa, Ae aegypti reaches 601 total individuals per liter in a tire in Senegal, An. gambiae 420 mosquitoes per liter in a puddle in Burkina Faso, and An. coluzzii 158 individuals per liter in a puddle in Mali. 
The population structure described by larval and pupal density parameters shows a high coefficient of variation (CV) within each species (Table 4). The respective densities of L1-2 larvae, L3-4 larvae and pupae for each site is highly variable across countries (Fig. 4), though Ae. albopictus MBS in Italy and Spain show comparable proportions of pupae (18\% and $20 \%$ respectively), and the proportions of late immature stages of $C_{x}$. pipiens MBS in Italy and Serbia are almost identical (12\% for pupae and $42 \%$ for L3-4). The overall range of mean pupal abundance falls between 5.5 to 12.8 pupae per liter across all species (Table 4 ). Culex pipiens in Europe, Ae. aegypti and the two Anopheles species in Africa display very similar pupal ratios (0.10-0.12), while Ae. albopictus populations show a proportion of pupae as twice as high and the lowest variation (Table 4).

\section{Discussion and conclusions}

We successfully tested harmonized protocols to characterize the ecoregional variation of MBS characteristics and productivity for five mosquito vectors of primary public health importance in Europe and Africa. The protocols effectively capture variations in the preference for MBS and associated water volume, larval and pupal densities and water temperature with interesting patterns across species.

The overlapping presence of $C x$. pipiens and Ae. albopictus in MBS with intermediate volume such as water barrels for irrigation and manholes highlights the widespread occurrence of larval competition in urbanized and rural environments (Carrieri et al. 2003). As both species infest small containers, niche partitioning for larval production and especially resource competition have been suggested (Carrieri et al. 2003, Marini et al. 2017, Müller et al 2018). Aedes aegypti preference for smaller sites $(<5 \mathrm{~L})$, typically tires and tree holes, shows wide habitat availability for congeneric Ae. albopictus as it expands its invasive range in Africa (Simard et al. 2005). In Senegal and Mali we detected An. gambiae populations in large puddles, and in Burkina in pools formed in clay quarries. This confirms the ability of the two Anopheles species to colonize a variety of aquatic habitats, regardless of permanence (Edillo et al. 2002, Fillinger et al. 2004, Gimmonneau 2014). One may speculate that An. gambiae and An. coluzzii habitat preference seems purely driven by availability of water bodies rather than other site characteristics such as volume and temperature. 
Interestingly, the range of mean water temperatures of MBS across species is small, between 26.3 and $27.6{ }^{\circ} \mathrm{C}$. This in vivo data on the range of large-scale temperature variation in MBS may improve the ability to design vector competence experiments in the laboratory, given that temperature during larval development is known to modulate vector competence (Westbrook et al. 2010, Murdock et al. 2014).

The variation of population densities and population structure is expectedly large within and across species inhabiting diverse MBS. The larval and pupal densities and their variation coincide with the density values reported for $C_{x}$. pipiens (Carrieri et al. 2013, Müller at al. 2018), Ae. albopictus (Eritja \& Herreros, 2017), Ae. aegypti (Kamgang et al. 2013) and the two Anopheles species (Edillo et al. 2004, Sogoba et al. 2007). Interestingly, the mean pupal densities of five species range between 5 and 12 per liter and we assume, that those numbers could feed well mathematical models describing vector population dynamics. Also the mean pupal ratios of four out of five species (except Ae. albopictus) are commensurably: pupae constitute $10-12 \%$ of all life-stages with the exception of $A e$. albopictus that shows a considerably higher pupal ratio, and the lowest variation. The unlike population structure and lower empirical variance might be related to the species adaptation as a colonizer of temporary waters. Optimal utilization of low resources leads to quicker larval development, which might skew Ae. albopictus population structure towards pupal stages (Marini et al. 2017), and lower predation pressure on immature stages in freshly colonized MBS could determine a higher overall MBS productivity.

As incidence of MBDs is globally rising, collaborative programs for insect vector surveillance at regional scale are crucial for predicting and managing outbreaks. Designing and implementing a multi-country mosquito vector monitoring program implies multiple organizational and ecoregional challenges. Limited manpower for professional field work in vector monitoring and control is a continuous challenge, thus reducing the temporal and geographical extent of areas monitored. In this study we demonstrate that collaborative projects among research groups are able to perform harmonized mosquito monitoring in two continents. We successfully employed harmonized protocols for characterizing mosquito MBS in six countries and over two continents. Thereby we confirmed that the available harmonized protocols represent a ready to use tool for implementing pan-regional vector surveillance programs and ecological studies. Our findings on population structures 
and densities might build a baseline for population modelling at continental scale for highly relevant mosquito vectors, and add important knowledge on species habitat preference and ecology that can be used in implementing IVM programs. Our results may also contribute to broaden the awareness of differences between the ways MBS are simulated in the laboratory against how they actually vary in the field. 


\section{Acknowledgments}

We thank Assétou Dionégué Diarra for data collection in Mali, Marta Verdun for technical support and data collection in Spain, and Dr Mihaela Kavran for technical assistance in Serbia, Marta Pagliochini for data entry, and Clause Meric for assistance with InfraVec web data download setup.

\section{Funding}

This project has received funding from the European Union's Horizon 2020 research and innovation programme Infravec2, Research infrastructures for the control of insect vectorborne diseases under grant agreement No 731060.

\section{References}

[1] Aranda, C., Martínez, M. J., Montalvo, T., Eritja, R., Navero-Castillejos, J., Herreros, E., ... \& Picart, L. (2018). Arbovirus surveillance: first dengue virus detection in local Aedes albopictus mosquitoes in Europe, Catalonia, Spain, 2015. Eurosurveillance, 23(47).

[2] Akiner, M. M., Demirci, B., Babuadze, G., Robert, V., \& Schaffner, F. (2016). Spread of the invasive mosquitoes Aedes aegypti and Aedes albopictus in the Black Sea region increases risk of chikungunya, dengue, and Zika outbreaks in Europe. PLoS neglected tropical diseases, 10(4), e0004664.

[3] Benelli, G., \& Mehlhorn, H. (2016). Declining malaria, rising of dengue and Zika virus: insights for mosquito vector control. Parasitology research, 115(5), 1747-1754.

[4] Bevins, S. N. (2008). Invasive mosquitoes, larval competition, and indirect effects on the vector competence of native mosquito species (Diptera: Culicidae). Biological Invasions, 10(7), 1109-1117.

[5] Carrieri, M., Bacchi, M., Bellini, R., \& Maini, S. (2003). On the competition occurring between Aedes albopictus and Culex pipiens (Diptera: Culicidae) in Italy. Environmental Entomology, 32(6), 1313-1321.

[6] Coetzee, M., Hunt, R. H., Wilkerson, R., Della Torre, A., Coulibaly, M. B., \& Besansky, N. J. (2013). Anopheles coluzzii and Anopheles amharicus, new members of the Anopheles gambiae complex. Zootaxa, 3619(3), 246-274. 
[7] Danis, K., Baka, A., Lenglet, A., Van Bortel, W., Terzaki, I., Tseroni, M., ... \& Dougas, G. (2011). Autochthonous Plasmodium vivax malaria in Greece, 2011. Eurosurveillance, 16(42), 19993.

[8] Derraik, J. G., \& Slaney, D. (2007). Anthropogenic environmental change, mosquitoborne diseases and human health in New Zealand. EcoHealth, 4(1), 72-81.

[9] De Wispelaere, M., Desprès, P., \& Choumet, V. (2017). European Aedes albopictus and Culex pipiens are competent vectors for Japanese encephalitis virus. PLoS neglected tropical diseases, 11(1), e0005294.

[10] ECDC (European Centre for Disease Prevention and Control), EFSA (European Food Safety Authority). (2018), Field sampling methods for mosquitoes, sandflies, biting midges and ticks - VectorNet project 2014-2018. ECDC and EFSA, Stockholm and Parma.

[11] Edillo, F. E., Touré, Y. T., Lanzaro, G. C., Dolo, G., \& Taylor, C. E. (2002). Spatial and habitat distribution of Anopheles gambiae and Anopheles arabiensis (Diptera: Culicidae) in Banambani village, Mali. Journal of Medical Entomology, 39(1), 70-77.

[12] Edillo, F. E., Touré, Y. T., Lanzaro, G. C., Dolo, G., \& Taylor, C. E. (2004). Survivorship and distribution of immature Anopheles gambiae sl (Diptera: Culicidae) in Banambani village, Mali. Journal of medical entomology, 41(3), 333-339.

[13] Eritja, Roger, and Eva Herreros. Spatial distribution of immature stages of Aedes albopictus (Skuse)(Diptera: Culicidae) in flower pots in a Spanish cemetery and field evaluation of metallic copper as a control agent. Journal of European Mosquito Control Association 35 (2017): 13-17.

[14] Fernandes, J. N., Moise, I. K., Maranto, G. L., \& Beier, J. C. (2018). Revamping mosquito-borne disease control to tackle future threats. Trends in parasitology, 34(5), 359-368.

[15] Fillinger, U., Sonye, G., Killeen, G. F., Knols, B. G., \& Becker, N. (2004). The practical importance of permanent and semipermanent habitats for controlling aquatic stages of Anopheles gambiae sensu lato mosquitoes: operational observations from a rural town in western Kenya. Tropical medicine \& international health, 9(12), 1274-1289.

[16] Gimonneau, G., Tchioffo, M. T., Abate, L., Boissière, A., Awono-Ambéné, P. H., Nsango, S. E., ... \& Morlais, I. (2014). Composition of Anopheles coluzzii and Anopheles gambiae microbiota from larval to adult stages. Infection, Genetics and Evolution, 28, 715-724. 
[17] Giron, S., Franke, F., Decoppet, A., Cadiou, B., Travaglini, T., Thirion, L., ... \& Noel, H. (2019). Vector-borne transmission of Zika virus in Europe, southern France, August 2019. Eurosurveillance, 24(45).

[18] Gubler, D. J. (1998). Resurgent vector-borne diseases as a global health problem. Emerging infectious diseases, 4(3), 442.

[19] Hotez, P.J. (2016). Neglected Tropical Diseases in the Anthropocene: The Cases of Zika, Ebola, and Other Infections. PLoS Negl Trop Dis 10(4): e0004648.

[20] Javelle, E., Florescu, S. A., Asgeirsson, H., Jmor, S., Eperon, G., Leshem, E., ... \& Eldin, C. (2019). Increased risk of chikungunya infection in travellers to Thailand during ongoing outbreak in tourist areas: cases imported to Europe and the Middle East, early 2019. Eurosurveillance, 24(10).

[21] Jones, K. E., Patel, N. G., Levy, M. A., Storeygard, A., Balk, D., Gittleman, J. L., \& Daszak, P. (2008). Global trends in emerging infectious diseases. Nature, 451(7181), 990.

[22] Kamgang, B., Ngoagouni, C., Manirakiza, A., Nakouné, E., Paupy, C., \& Kazanji, M. (2013). Temporal patterns of abundance of Aedes aegypti and Aedes albopictus (Diptera: Culicidae) and mitochondrial DNA analysis of Ae. albopictus in the Central African Republic. PLoS neglected tropical diseases, 7(12).

[23] Lanzaro, G. C., \& Lee, Y. (2013). Speciation in Anopheles gambiae-The distribution of genetic polymorphism and patterns of reproductive isolation among natural populations. In Anopheles mosquitoes-New insights into malaria vectors. IntechOpen.

[24] Marini, G., Calzolari, M., Angelini, P., Bellini, R., Bellini, S., Bolzoni, L., ... \& Pugliese, A. (2020). A quantitative comparison of West Nile virus incidence from 2013 to 2018 in Emilia-Romagna, Italy. PLoS Neglected Tropical Diseases, 14(1).

[25] Marini, G., Guzzetta, G., Baldacchino, F., Arnoldi, D., Montarsi, F., Capelli, G., ... \& Rosà, R. (2017). The effect of interspecific competition on the temporal dynamics of Aedes albopictus and Culex pipiens. Parasites \& vectors, 10(1), 102.

[26] Medlock, J., Balenghien, T., Alten, B., Versteirt, V., \& Schaffner, F. (2018). Field sampling methods for mosquitoes, sandflies, biting midges and ticks: VectorNet project 2014-2018. EFSA Supporting Publications, 15(6), 1435E.

[27] Murdock, C. C., Blanford, S., Luckhart, S., \& Thomas, M. B. (2014). Ambient temperature and dietary supplementation interact to shape mosquito vector competence for malaria. Journal of insect physiology, 67, 37-44. 
[28] Müller, R., Knautz, T., Vollroth, S., Berger, R., Kreß, A., Reuss, F., Groneberg, D.A., Kuch, U. (2018) Larval superiority of Culex pipiens to Aedes albopictus in a replacement series experiment: prospects for coexistence in Germany. Parasites \& Vectors, 11, 80

[29] Okech, B. A., Gouagna, L. C., Yan, G., Githure, J. I., \& Beier, J. C. (2007). Larval habitats of Anopheles gambiae ss (Diptera: Culicidae) influences vector competence to Plasmodium falciparum parasites. Malaria journal, 6(1), 50.

[30] Okogun, G. R., Anosike, J. C., Okere, A., Nwoke, B., \& Esekhegbe, A. (2003). Epidemiological implications of preferences of breeding sites of mosquito speciesin Midwestern Nigeria. Annals of Agricultural and Environmental Medicine, 10(2), 217-222.

[31] Pascoe, E. L., Pareeth, S., Rocchini, D., \& Marcantonio, M. (2019). A Lack of "Environmental Earth Data" at the Microhabitat Scale Impacts Efforts to Control Invasive Arthropods That Vector Pathogens. Data, 4(4), 133.

[32] Sarma, N., Ullrich, A., Wilking, H., Ghozzi, S., Lindner, A. K., Weber, C., ... \& VygenBonnet, S. (2018). Surveillance on speed: Being aware of infectious diseases in migrants mass accommodations-an easy and flexible toolkit for field application of syndromic surveillance, Germany, 2016 to 2017. Eurosurveillance, 23(40).

[33] Schneider, J. R., Chadee, D. D., Mori, A., Romero-Severson, J., \& Severson, D. W. (2011). Heritability and adaptive phenotypic plasticity of adult body size in the mosquito Aedes aegypti with implications for dengue vector competence. Infection, Genetics and Evolution, 11(1), 11-16.

[34] Simard, F., Nchoutpouen, E., Toto, J. C., \& Fontenille, D. (2005). Geographic distribution and breeding site preference of Aedes albopictus and Aedes aegypti (Diptera: Culicidae) in Cameroon, Central Africa. Journal of medical entomology, 42(5), 726-731.

[35] Sogoba, N., Doumbia, S., Vounatsou, P., Baber, I., Keita, M., Maiga, M., ... \& Ribeiro, J. M. (2007). Monitoring of larval habitats and mosquito densities in the Sudan savanna of Mali: implications for malaria vector control. The American journal of tropical medicine and hygiene, 77(1), 82-88.

[36] Vaux, A. G., Murphy, G., Baskerville, N., Burden, N., Convery, N., Crossley, L., ... \& Napier, K. (2011). Monitoring for invasive and endemic mosquitoes at UK ports. Eur. Mosq. Bull, 29, 133-140. 
[37] Vernick, K. D. (2017). Infravec2: expanding researcher access to insect vector tools and resources. Pathogens and global health, 111(5), 217.

[38] Weaver, S. C., Charlier, C., Vasilakis, N., \& Lecuit, M. (2018). Zika, chikungunya, and other emerging vector-borne viral diseases. Annual review of medicine, 69, 395-408.

[39] Vallorani, R., Angelini, P., Bellini, R., Carrieri, M., Crisci, A., Mascali Zeo, S., ... \& Venturelli, C. (2015). Temperature characterization of different urban microhabitats of Aedes albopictus (Diptera Culicidae) in central-northern Italy. Environmental Entomology, 44(4), 1182-1192.

[40] Versteirt, V., Alten, B., Balenghien, T., Medlock, J., \& Schaffner, F. (2017). VectorNet: a pan-European approach towards a standardized data collection on distribution and abundance of vectors of medical and veterinary importance.

[41] Westbrook, C. J., Reiskind, M. H., Pesko, K. N., Greene, K. E., \& Lounibos, L. P. (2010). Larval environmental temperature and the susceptibility of Aedes albopictus Skuse (Diptera: Culicidae) to Chikungunya virus. Vector-Borne and Zoonotic Diseases, 10(3), 241-247.

[42] Winters, A. M., Bolling, B. G., Beaty, B. J., Blair, C. D., Eisen, R. J., Meyer, A. M., ... \& Eisen, L. (2008). Combining mosquito vector and human disease data for improved assessment of spatial West Nile virus disease risk. The American journal of tropical medicine and hygiene, 78(4), 654-665

[43] Whitty, C. J., \& Ansah, E. (2019). Malaria control stalls in high incidence areas. BMJ, $365,12216$. 
Graziosi et al. 2020. Tables 1-4

Table 1. Mosquito breeding sites within a six-country survey conducted in Europe and Africa across different landscapes.

\begin{tabular}{llccccc}
\hline \multirow{2}{*}{ Species } & Country & Urban & Urban & Rural & Natural & Total \\
\hline Cx. pipiens & Spain & 8 & - & 9 & 4 & 21 \\
& Italy & 6 & 6 & 6 & 6 & 24 \\
& Serbia & 8 & - & 8 & 8 & 24 \\
Ae. albopictus & Spain & 8 & - & 9 & - & 17 \\
& Italy & 6 & 6 & 6 & - & 18 \\
Ae. aegypti & Senegal & 6 & - & 6 & 7 & 19 \\
An. gambiae & Senegal & 8 & - & 6 & - & 14 \\
& Burkina Faso & 12 & 6 & 12 & 6 & 36 \\
An. coluzzii & Mali & 6 & 6 & 6 & - & 18 \\
\hline \hline & In total & 68 & 24 & 68 & 31 & 191 \\
& \% & 35.6 & 12.6 & 35.6 & 16.2 & 100 \\
\hline \hline
\end{tabular}


Table 2. Typology of Mosquito breeding sites (MBS) of five target mosquito species including range of water volume (6 classes) as surveyed across six countries at two continents. The number of most common MBS types per species is marked in bold.

\begin{tabular}{|c|c|c|c|c|c|c|c|c|c|c|}
\hline \multirow[b]{2}{*}{ Aquatic habitat } & \multirow{2}{*}{$\begin{array}{l}\text { Volume class }^{1} \\
\begin{array}{llllll}1 & 2 & 3 & 4 & 5 & 6\end{array}\end{array}$} & \multicolumn{3}{|c|}{ Culex pipiens } & \multicolumn{2}{|c|}{$\begin{array}{c}\text { Ae. } \\
\text { albopictus }\end{array}$} & $\begin{array}{c}\text { Ae. } \\
\text { aegypti }\end{array}$ & \multicolumn{2}{|c|}{ An. gambiae } & \multirow{2}{*}{$\begin{array}{c}\text { An. } \\
\text { coluzzi } \\
\text { Mali }\end{array}$} \\
\hline & & Spain & Italy & Serbia & Spain & Italy & & & Burkina & \\
\hline Pot & & - & - & - & 7 & 2 & 6 & - & - & - \\
\hline Tire & & - & - & - & - & - & 7 & - & - & - \\
\hline Tree hole & & - & - & - & - & - & 6 & - & - & - \\
\hline Puddle & & - & - & - & - & - & - & 13 & 4 & 18 \\
\hline Bucket & & - & 1 & 1 & 4 & 3 & - & - & - & - \\
\hline Manhole/scupper & & 3 & 8 & - & 1 & 6 & - & - & - & - \\
\hline Rainwater barrel & & 3 & 2 & 1 & 5 & 7 & - & - & - & - \\
\hline Clay quarry & & - & - & - & - & - & - & 1 & 26 & - \\
\hline Ditch & & - & 8 & - & - & - & - & - & - & - \\
\hline Canal & & 1 & - & 13 & - & - & - & - & 6 & - \\
\hline Pond & & 6 & 3 & - & - & - & - & - & - & - \\
\hline Stream/river pool & & 8 & 2 & - & - & - & - & - & - & - \\
\hline Wetland/swamp & & - & - & 9 & - & - & & - & - & - \\
\hline
\end{tabular}

${ }^{1}$ Volume classes: 1 (<0.5 L), 2 (0.5-5.0 L), 3 (5-50 L), 4 (50-200 L), 5 (200-2000 L), 6 (>2000 L) 
Table 3. Overall variation of water temperature $\left[{ }^{\circ} \mathrm{C}\right]$ was measured for the target species $C x$. pipiens, Ae. albopictus, Ae. aegypti, An. gambiae and An. coluzzii in each mosquito breeding site. Next to the mean value per species, the overall variation is given as standard deviation (STD), coefficient of variation (CV), and minimum (Min) and maximum (Max) values for each species.

\begin{tabular}{|c|c|c|c|c|c|}
\hline & $\begin{array}{c}\text { Cx. } \\
\text { pipiens }\end{array}$ & $\begin{array}{c}\text { Ae. } \\
\text { albopictus }\end{array}$ & $\begin{array}{c}\text { Ae. } \\
\text { aegypti }\end{array}$ & $\begin{array}{c}\text { An. } \\
\text { gambiae }\end{array}$ & $\begin{array}{c}\text { An. } \\
\text { coluzzii }\end{array}$ \\
\hline \multicolumn{6}{|c|}{ Water temp. ${ }^{\circ} \mathrm{C}$} \\
\hline MEAN & 26.3 & 27.6 & 27.3 & 26.7 & 27.0 \\
\hline STD & 4.1 & 3.7 & 1.6 & 2.6 & 2.2 \\
\hline CV \% & 15.7 & 13.3 & 5.8 & 9.9 & 8.0 \\
\hline Min & 20.2 & 19.7 & 24.8 & 22 & 23 \\
\hline Max & 37.6 & 37.1 & 31.3 & 31.8 & 30 \\
\hline
\end{tabular}


Table 4. The total number of aquatic life stages [mean ( $\mathrm{N}$ larvae + pupae per liter)] and respective pupal ratio ( $\mathrm{N}$ pupae / total $\mathrm{N}$ ) per Mosquito breeding sites (MBS) were calculated for the target species Cx. pipiens Ae. albopictus, Ae. aegypti, An. gambiae and An. coluzzii. Together with the mean value per species, the overall variation among MBS is given as standard deviation (STD), coefficient of variation (CV), and minimum (Min) and maximum (Max) values for each species.

\begin{tabular}{|c|c|c|c|c|c|}
\hline & $\begin{array}{c}C x . \\
\text { pipiens }\end{array}$ & $\begin{array}{c}\text { Ae. } \\
\text { albopictus }\end{array}$ & $\begin{array}{c}\text { Ae. } \\
\text { aegypti }\end{array}$ & $\begin{array}{c}\text { An. } \\
\text { gambiae }\end{array}$ & $\begin{array}{c}\text { An. } \\
\text { coluzzii }\end{array}$ \\
\hline \multicolumn{6}{|c|}{ Larval density (L1-2) } \\
\hline MEAN & 71.8 & 19.1 & 41.4 & 55.1 & 38.6 \\
\hline STD & 93.6 & 27.1 & 99.1 & 76.3 & 20.7 \\
\hline $\mathrm{CV} \%$ & 130.4 & 142.1 & 239.2 & 138.6 & 53.7 \\
\hline Min & 0 & 0 & 0 & 0 & 10 \\
\hline Max & 377 & 107 & 422 & 397 & 76.7 \\
\hline \multicolumn{6}{|c|}{ Larval density (L3-4) } \\
\hline MEAN & 58.5 & 36.8 & 46.6 & 23.3 & 45.6 \\
\hline STD & 92.0 & 9.8 & 101.4 & 22.9 & 22.8 \\
\hline CV \% & 157.2 & 26.6 & 217.4 & 98.4 & 50.1 \\
\hline Min & 0 & 0.2 & 0 & 0 & 6 \\
\hline Max & 475 & 118 & 430 & 96.0 & 85 \\
\hline \multicolumn{6}{|c|}{ Pupal density } \\
\hline MEAN & 12.8 & 8.9 & 5.5 & 7.4 & 8.6 \\
\hline STD & 27.7 & 9.8 & 8.8 & 5.5 & 10.6 \\
\hline $\mathrm{CV} \%$ & 216.4 & 109.6 & 160.5 & 74.1 & 122.2 \\
\hline Min & 0.5 & 0.6 & 0 & 0 & 1 \\
\hline Max & 204 & 33.5 & 26 & 25.0 & 40 \\
\hline \multicolumn{6}{|c|}{ Total population } \\
\hline MEAN & 143.0 & 46.8 & 93.5 & 85.8 & 92.8 \\
\hline STD & 180.1 & 40.4 & 175.4 & 91.8 & 42.4 \\
\hline CV \% & 125.9 & 86.3 & 187.6 & 106.9 & 45.7 \\
\hline Min & 3 & 5 & 1 & 3 & 30 \\
\hline Max & 928 & 123 & 601 & 420 & 157 \\
\hline \multicolumn{6}{|c|}{ Pupal ratio } \\
\hline MEAN & 0.12 & 0.26 & 0.12 & 0.10 & 0.11 \\
\hline STD & 0.14 & 0.20 & 0.26 & 0.09 & 0.14 \\
\hline CV \% & 117.1 & 79.0 & 211.0 & 86.5 & 123.7 \\
\hline Min & 0.001 & 0.002 & 0 & 0 & 0.01 \\
\hline Max & 1 & 0.90 & 0.29 & 0.33 & 0.55 \\
\hline
\end{tabular}




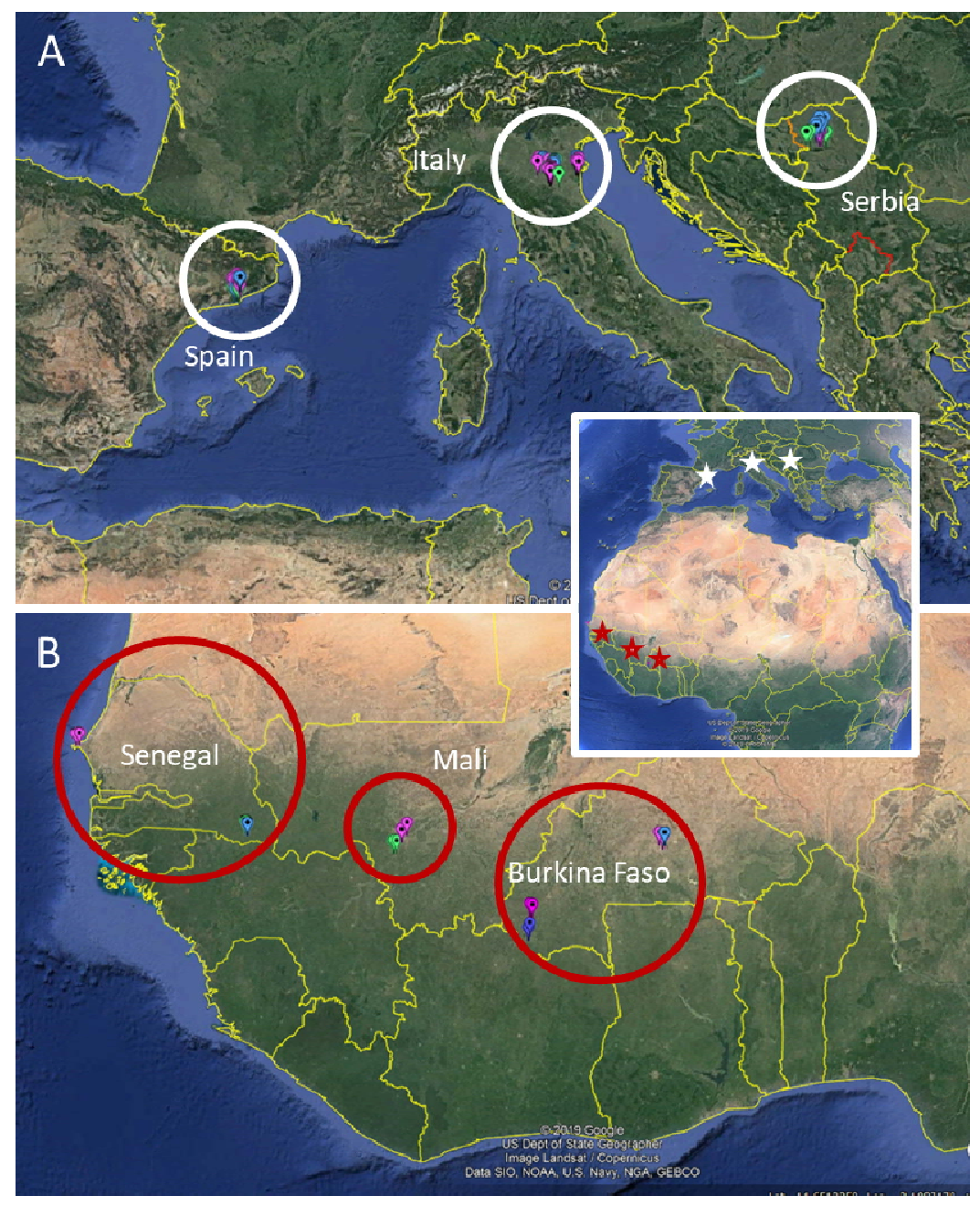

Fig. 1. Harmonized mosquito monitoring protocols were tested in six countries located in: A) Europe (Spain, Italy, Serbia) and B) Sub-Saharan Africa (Senegal, Burkina Faso and Mali). (Imagery: Google Earth). Locations of mosquito breeding sites are available for download at: https://infravec2.eu/mosquito-survey-data/ 
A. Europe. Cx. pipiens

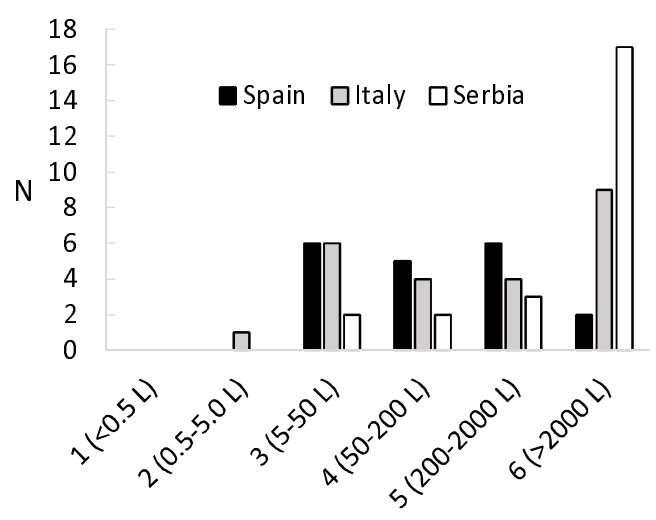

C. Africa. Ae. aegypti

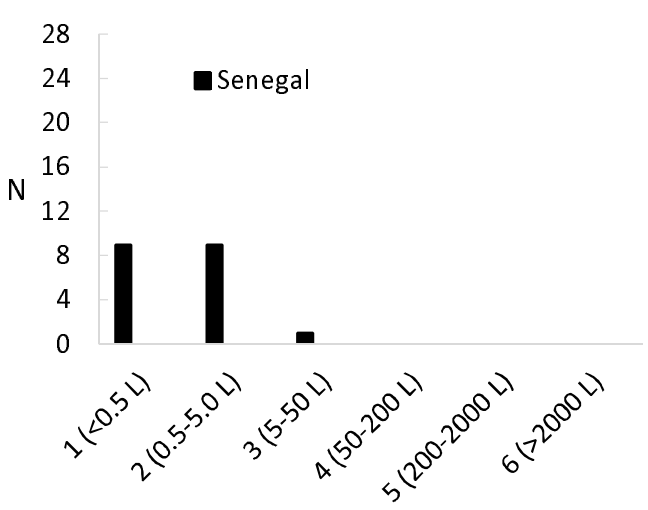

B. Europe. Ae. albopictus

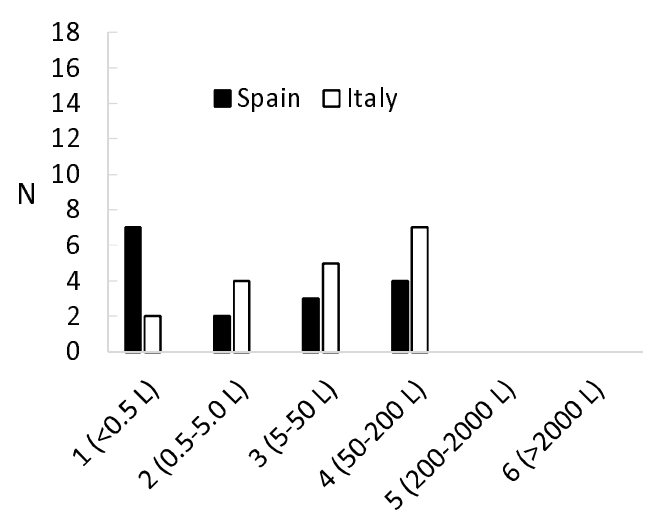

D. Africa. An. gambiae, An.coluzzii

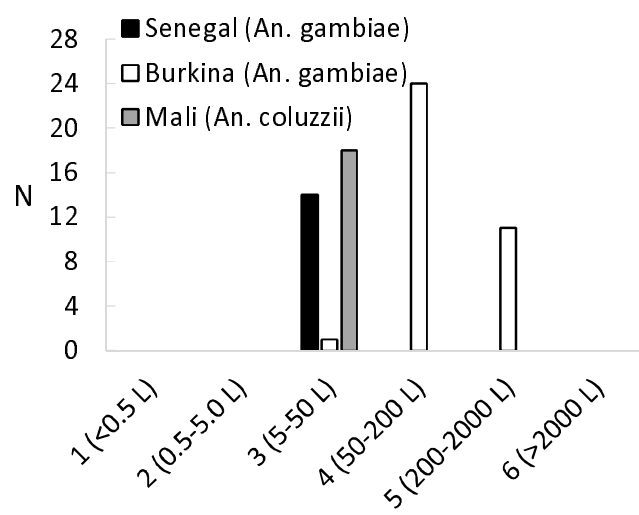

Fig. 2. Number of aquatic individuals (larvae and pupae) per liter for five target mosquito species in six water volume classes of larval production sites as surveyed across six countries at two continents: $\mathrm{Cx}$. pipiens (A), Ae. albopictus (B), Ae. aegypti (C), An. gambiae in Burkina Faso and Senegal and An. coluzzii in Mali (D). 


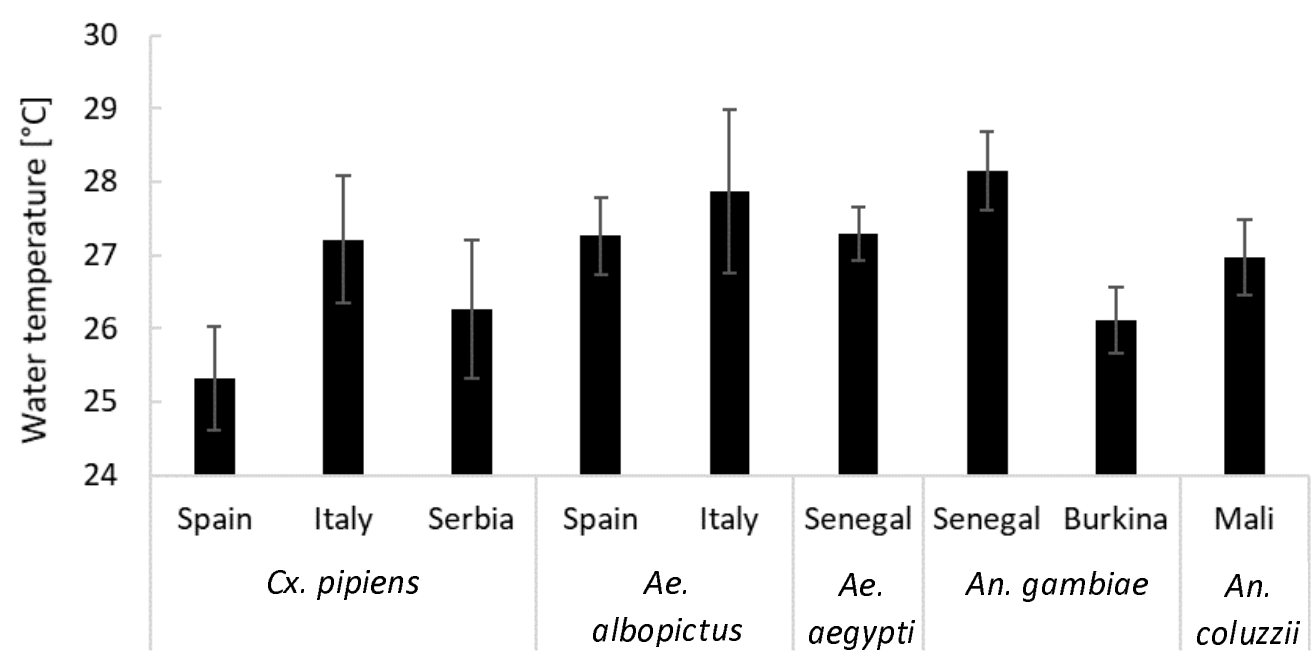

Fig. 3 Water temperature $\left[{ }^{\circ} \mathrm{C} \pm\right.$ standard error] for five target mosquito species across six surveyed countries at two continents. Surveys were completed during peak season in European countries (July and August) and in Senegal and Burkina Faso (September to November), and at the end of the peak season in Mali (December). 


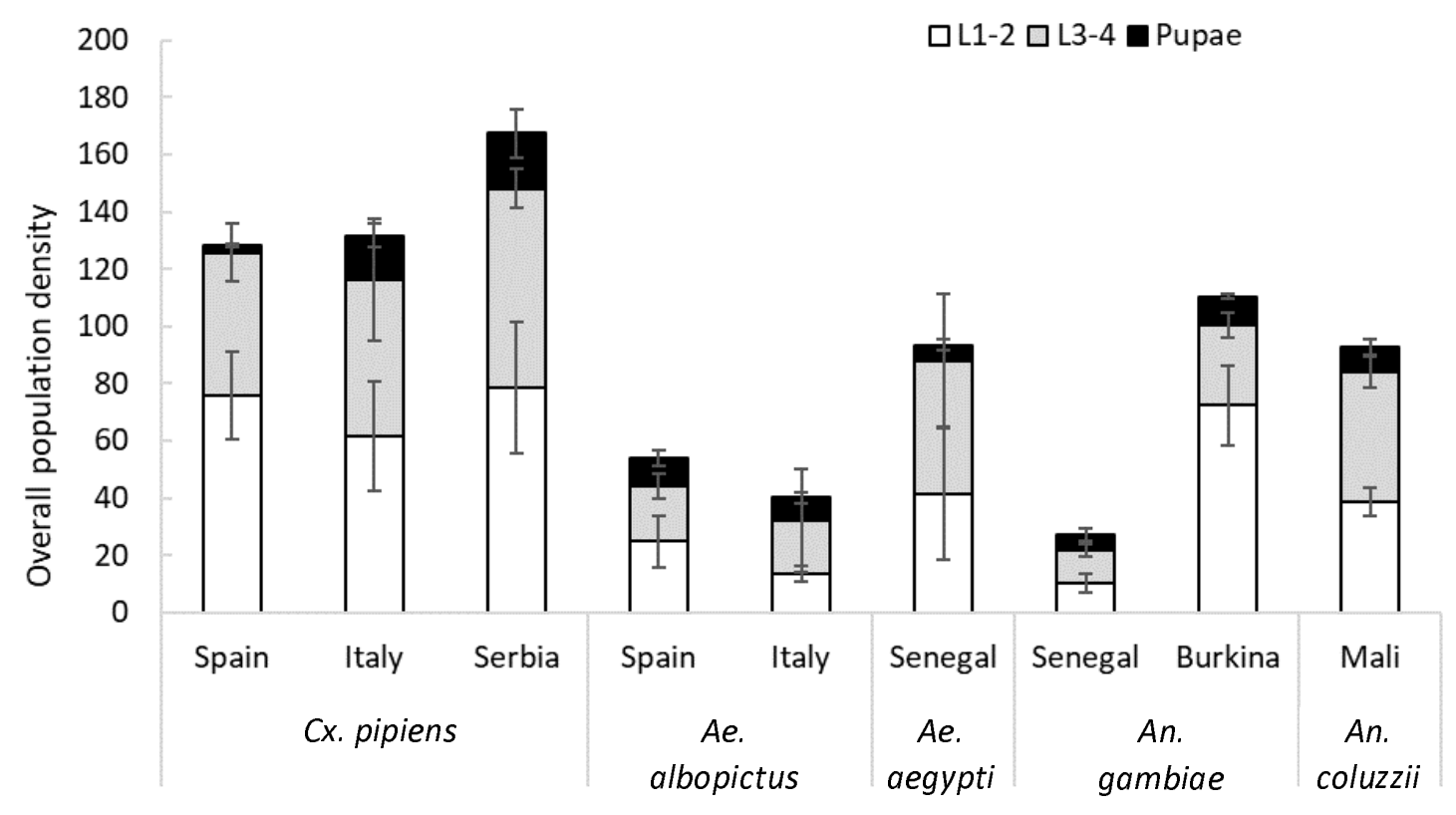

Fig. 4. Larval (L1-2 and L3-4) and pupal densities per liter (mean \pm standard error) for five target mosquito species surveyed in six countries at two continents. Surveys were completed during the peak season in European countries (July and August) and in Senegal and Burkina Faso (September to November), and at the end of the peak season in Mali (December). 